\title{
Evaluation of an ICD logging system to supplement an EMR in a Sub-Saharan country
}

\author{
Araba A. Wubah ${ }^{1}$, Jean A. Yankson ${ }^{2}$, Cameron Sumpter ${ }^{1}$, Fred Rawlins ${ }^{3}$, Dean Sutphin ${ }^{4}$, Kim Menier $^{5}$, Harold R. \\ Garner*1,6 \\ ${ }^{1}$ Center for Bioinformatics and Genetics and the Primary Care Research Network, Edward Via College of Osteopathic Medicine \\ (VCOM), Blacksburg, VA, United States \\ ${ }^{2}$ Healthwise Medical Center, Greater Accra Region, Ghana, United States \\ ${ }^{3}$ Simulation and Technology Center, VCOM, United States \\ ${ }^{4}$ International and Appalachian Outreach, VCOM, United States \\ ${ }^{5}$ Heliotext, Blacksburg, VA, United States \\ ${ }^{6}$ Gibbs Cancer Center and Research Institute, Spartanburg, SC, United States
}

Received: January 11, 2018

DOI: $10.5430 /$ jha.v7n2p8
Accepted: February 22, $2018 \quad$ Online Published: February 27, 2018

URL: https://doi.org/10.5430/jha.v7n2p8

\begin{abstract}
Adoption of electronic medical records (EMRs) has been spotty and sluggish in the world, including the United States, despite the multiple benefits of medical technology and informatics. Though there are difficulties in establishing and maintaining an EMR system in a developing country, it is not impossible. The International Classification of Diseases (ICD) Logger, called CREDO (Clinical Rotation Evaluation and Documentation Organizer), developed by Edward Via College of Osteopathic Medicine (VCOM), provides a straightforward, economical EMR system to use in a developing country, such as Ghana. However, with a recently established EMR system developed locally and being used at the target new hospital, Healthwise Medical Center, the aim of the study was to use the common medical documentation language of the World Health Organization (WHO) ICD-10 codes to add value to the local EMR. This demonstration enabled the comparison of medical encounters in Ghana to those in the United States, specifically in Appalachia where VCOM students typically do their clinical rotations. We also evaluated the issues and tested the CREDO ICD Logger as a simple, stand-alone EMR system. Therefore, by collecting ICD data twice weekly from Ghana, a data point in Sub-Saharan Africa, it became possible to compare a public health snapshot of developing countries and sites in the United States.
\end{abstract}

Key Words: International Classification of Diseases, Logger, Electronic medical records, Medical informatics

\section{INTRODUCTION}

The electronic medical record (EMR) has the potential to advance medical technology and care to unprecedented heights by consolidating patients' data to a central location, tracking the patients over time and providing preventative care, such as screenings or checkups. ${ }^{[1]}$ Adoption of EMRs has been steadily increasing in the United States, with a $60 \%$ increase in use from 2001 to 2013. ${ }^{[2]}$ At present, nearly $90 \%$ of office based physicians use EMRs and about $67 \%$ of all providers use some form of EMRs. ${ }^{[3]}$ However, many physicians still struggle with exploiting the full potential of the EMR, due to insufficient training, lack of user-friendliness and the trade-

*Correspondence: Harold R. Garner; Email: skipgarner@gmail.com; Address: Center for Bioinformatics and Genetics and the Primary Care Research Network, Edward Via College of Osteopathic Medicine (VCOM), Blacksburg, VA, United States. 
off between interfacing with the system or spending time with the patient. ${ }^{[2,4,5]}$

Some researchers and developers have moved to sharing their software and hardware with developing countries. Though there are many difficulties in establishing and maintaining an EMR in a developing country, it is not impossible. This is proven true by examples of systems built locally, within developing countries, and internationally with the aid of the United States. These systems are currently being used in a range of medical infrastructure environments, from general hospitals to small polyclinics. ${ }^{[6]}$ While the benefits of having an EMR system are plentiful, the hindrances can be just as numerous.

Electronic documentation of patients' information is typically one of the first noted advantages of EMR systems. Concerns about the conditions of paper documents being worn down by nature's elements and time, especially in developing countries without adequate storage facilities, are abated by EMRs. Patients' diagnoses and prescriptions can be electronically stored and backed-up on other servers, if necessary, and easily searched and summarized. However, security quickly becomes a critical issue. Although hacking of medical records is not a common issue within Ghana, it is not unusual for computers to be left on and unattended with patient information easily accessible for inappropriate use. There are also issues with incomplete, inaccurate or unreliable data, due to improper staff training or lack of investment in patient confidentiality, which could hinder the progress of an EMR in a developing country. ${ }^{[7]}$

Occasionally, an EMR system may require an internet connection to allow storage of information in a cloud server. Ghana does have internet capabilities, but connectivity is often sporadic. For a well-established company, like the Pan African bank, Ecobank, internet connection, speed and strength is a necessity for a banking conglomerate (and they are able to secure this due to their size and established IT security team). But for a smaller company or establishment, internet must frequently be purchased through a contract with a telecommunications company, such as Vodafone or MTN, and at times may not be as powerful or reliable as needed. While a web-based system provides a stable source of information management for EMR systems, the power and network connectivity can affect overall performance and convenience. ${ }^{[8,9]}$

In terms of the data used in EMR systems, the International Classification of Diseases (ICD) is the standard diagnostic documentation tool for clinical purposes mandated by the World Health Organization. ${ }^{[10]}$ Currently, 117 countries use the tenth revision of the ICD (ICD-10); an eleventh re- vision will be released in the next year. Using this as its foundation, the CREDO ICD Logger was built to aid thirdand fourth-year medical students at the Edward Via College of Osteopathic Medicine (VCOM) in capturing their clinical experience through logging patient encounter associated ICD-10 codes, including information gathered by conventional EMR systems. ${ }^{[11]}$ As most medical curricula do not provide a course on medical informatics, faculty at VCOM developed this program to aid its students. The design is simple and intuitive, with most users mastering it within ten minutes. It provides diagnosis, procedure and pharmaceutical pages that allow the students to enter the respective codes, minimal demographic information (i.e., age and gender) and free-text notes, without divulging any personally identifying information.

The aim of this study was to investigate and implement the use of the CREDO ICD Logger into a hospital in a developing country to benefit their data warehousing and organizational practices. Given that the Healthwise Medical Center (HMC) was somewhat advanced in comparison to other hospitals in Ghana with its own custom EMR system, our goal was to supplement that EMR system with universal ICD-10 codes to enable the comparison of public health demographics locally to other world sites. The CREDO ICD Logger contains no personal identifying information, relying on a unique internal patient ID. This means that data can be shared without concerns regarding patient privacy, including adhering to HIPAA regulations (in the US). Furthermore, it provides a way to contribute this anonymous data for public health comparisons as well as for off-site backup of patient records. To ensure privacy, patient data is securely stored in a local confidential database that connects the CREDO ICD Logger patient ID (a number) to the true patient identifiers.

\section{Methods}

\subsection{Setting}

HMC is a private hospital located in Community 1 of Tema in the Greater Accra Region of Ghana, $30 \mathrm{~km}$ east of Accra. The capital city, Accra, has a population of approximately 2 million; the harbor city of Tema's population is approximately $156,000 .^{[12]}$ This medical center is part of the Ghana Wellness Foundation, which primarily provides free health screenings for local residents every few months. ${ }^{[13]}$ The hospital is in a prime location to receive patients, due to its proximity to one of the two main harbors in Ghana. HMC began seeing patients in January 2017, although in limited quantities and for limited service because of a lack of medical instruments. In April, after receiving instruments from Project C.U.R.E., a 501(c)(3) non-profit organization that provides medical supplies and equipment to developing coun- 
tries, HMC had a soft opening, allowing patients with certain types of health insurance and those willing to pay out of pocket to come to the hospital. As of August 2017, HMC has its main site located in Tema and two satellite sites located at the harbor approximately $5 \mathrm{~km}$ south and a quarry located in Shai Hills about $30 \mathrm{~km}$ northeast. The satellite sites provide clinical services to a construction company in partnership with HMC.

\subsection{Design}

The CREDO ICD Logger is a simple and intuitive Web-based system that is primarily used for educational purposes at VCOM. ${ }^{[11]}$ Using the 10th revision of the International Statis- tical Classification of Diseases and Related Health Problems (ICD-10) and the World Health Organization Anatomical Therapeutic Chemical (WHO-ATC) Classification System, the CREDO ICD Logger captures the most current diagnoses, procedures and medications in a way that is universal. It is also HIPAA-compliant, in that it only requires the most basic information about patients without divulging personally identifying information (see Figure 1). There is the Logger page, where users can log patient diagnoses, procedures and prescriptions, and then the Dashboard, where users can view and search through the entries that they have made as well as generate appropriate reports.

\begin{tabular}{|c|c|c|c|c|c|c|c|c|}
\hline Patient Number & First Name & Last Name & Gender & Birthday & \multicolumn{3}{|c|}{ Local Address } & ICD Diagnos is \\
\hline 29173 & Alberta & Danso & Female & $9 / 1 / 17$ & \multicolumn{3}{|c|}{ Sunyani, Brong Ahafo Region } & P14.9 \\
\hline 29175 & Moses & Oteng & Male & $1 / 6 / 85$ & \multicolumn{3}{|c|}{ Box GP 3338, Accra } & W36.3; S85.912 \\
\hline 29176 & Kwame & Prempeh & Male & $11 / 4 / 57$ & \multicolumn{3}{|c|}{ No. 25 Kanfla Street, Asylum Down, Accra } & K42.9 \\
\hline 29188 & Prosper & Ogyampah & Female & $8 / 8 / 95$ & \multicolumn{3}{|c|}{ Boukrom, Near Airport Roundabout, Kumasi } & Z59.4 \\
\hline 29191 & Michael & Ekuoba & Male & $4 / 17 / 17$ & \multicolumn{3}{|c|}{ S/R BO12, McCarthy Hill, Accra } & H21.01 \\
\hline 29192 & Kodjo & Williams & Male & $7 / 27 / 76$ & \multicolumn{3}{|c|}{ No. 10, First Anege Link, Dansoman, Accra } & C23 \\
\hline 29194 & Benjamin & Twum & Male & $2 / 16 / 45$ & \multicolumn{3}{|c|}{501 Kwame Nkrumah Ave., Adabraka, Accra } & N22 \\
\hline 29198 & Eleanor & Ofori & Female & $2 / 17 / 06$ & \multicolumn{3}{|c|}{ P.O. Box LA 149, Accra } & Y27.3; T23.001 \\
\hline 29231 & Kwabena & Oppong & Male & $7 / 29 / 49$ & \multicolumn{3}{|c|}{ Aviation Road Airport Residential Area, Accra } & F03.91 \\
\hline 29263 & Ebeneezer & Mensah & Male & $12 / 4 / 60$ & \multicolumn{3}{|c|}{ P.O. Box C2141 Cantonments, Accra } & J63.3 \\
\hline 29265 & Felicity & Boateng & Female & $6 / 3 / 75$ & \multicolumn{3}{|c|}{ P.O. BOX KN 854, Accra } & R65.20 \\
\hline 29932 & Ama & Ansah & Female & $9 / 7 / 62$ & \multicolumn{3}{|c|}{ Plot 7A Block A, Accra } & H40.051 \\
\hline \multicolumn{9}{|l|}{ ICD LOGGER } \\
\hline$\circ \mathrm{c}$ & de & Description & & $\circ$ & Patient & Rotation & $\odot$ Location & $\circ$ Date \\
\hline \multicolumn{2}{|c|}{ S85.912 } & $\begin{array}{l}\text { Laceration of unspec } \\
\text { level, left leg }\end{array}$ & fied blood vesse & el at lower leg & 19175 & [127] ICD Development & vcom Virginia & 2017-09-06 13:58:11 \\
\hline \multicolumn{2}{|c|}{ H40.051 } & Ocular hypertension, & ight eye & & 29932 & [127] ICD Development & vcom Virginia & 2017-09-06 13:31:55 \\
\hline \multicolumn{2}{|c|}{ T23.001 } & \multicolumn{3}{|c|}{$\begin{array}{l}\text { Burn of unspecified degree of right hand, unspecified } \\
\text { site }\end{array}$} & 29198 & [127] ICD Development & vcom Virginia & 2017-09-05 17:09:48 \\
\hline \multicolumn{2}{|c|}{ R65.20 } & \multicolumn{3}{|c|}{ Severe sepsis without septic shock } & 29265 & [127] ICD Development & vcom Virginia & 2017-09-05 17:02:12 \\
\hline \multicolumn{2}{|c|}{ J63.3 } & \multicolumn{3}{|c|}{ Graphite fibrosis (of lung) } & 29263 & [127] ICD Development & vcom Virginia & 2017-09-05 16:58:52 \\
\hline \multicolumn{2}{|c|}{ F03.91 } & \multicolumn{3}{|c|}{ Unspecified dementia with behavioral disturbance } & 29231 & [127] ICD Development & vcom Virginia & 2017-09-05 16:31:44 \\
\hline \multicolumn{2}{|c|}{ Y27.3 } & \multicolumn{3}{|c|}{$\begin{array}{l}\text { Contact with hot household appliance, undetermined } \\
\text { intent }\end{array}$} & 29198 & [127] ICD Development & vcom Virginia & 2017-09-05 15:36:42 \\
\hline \multicolumn{2}{|c|}{ N22 } & \multicolumn{3}{|c|}{$\begin{array}{l}\text { Calculus of urinary tract in diseases classified } \\
\text { elsewhere }\end{array}$} & 29194 & [127] ICD Development & vcom Virginia & 2017-09-05 15:35:23 \\
\hline \multicolumn{2}{|c|}{$\mathrm{C23}$} & \multicolumn{3}{|c|}{ Malignant neoplasm of gallbladder } & 29192 & [127] ICD Development & vcom Virginia & 2017-09-05 15:32:56 \\
\hline 70411 & 21.01 & Hyphema, right eye & & & 29191 & [127] ICD Development & vcom Virginia & 2017-09-05 15:32:07 \\
\hline
\end{tabular}

Figure 1. An Excel table with pseudo-patient's information and the CREDO ICD Logger output table of specific diagnoses. Patients are highlighted to show the correspondence between the local Excel spreadsheet information and the Logger's Patient Number (ID) 
The welcome screen has tabs that lead to the two pages. On the left side is where the user can access their account or $\log$ out, if necessary. On the right side, there are links to the support team, user instructions, FAQ documents and a brief "about" page. On the logging page, the user has the option of allowing geolocation to be activated, to provide better data collection for the development team's use. From there, the user can navigate between the Diagnosis, Procedures, and Drug pages. In the Diagnosis page, users can enter ICD $10-\mathrm{CM}$ codes by selecting categories, typing in a diagnosis or synonyms, searching the top 10 diagnoses or choosing the most recent diagnosis entries. Users also log whether the patient is new or is an existing one, the gender and age, and finally enter any notes that do not disclose confidential information. In the Procedures page, like the Diagnosis page users can enter the ICD-10-PCS code through the four different methods and enter the demographic information. They can also include the engagement level, either "Observed" or "Performed", which is primarily for the medical students. In the Drug page, users can enter the WHO-ATC code through the four methods and enter the demographic information. Dosages can be placed in the notes section. On the Dashboard page, users can see how many other users are using the Logger, their own statistics of logging and then all the entries they have made. If necessary, they may edit information by clicking on an entry. Users can also export information into spread sheets, charts and tables, filtered to their needs to aid in creating reports.

A locally made spreadsheet which incorporates patient identifiers obtained from the local EMR system, HealthMaster program, was created to associate personal contact information to the CREDO ICD Logger Internal Patient Number (identifier), thereby making a link to the software and the web-based program. The spreadsheet is encrypted, in order to prevent confidential information from being exposed, and only authorized personnel may access it. The spreadsheet allows clinicians to make new or review ICD entries or information for a specific patient via the CREDO ICD Logger Internal Patient Number. The Logger provides anonymous patient specific data, which does not violate Ghana's confidentiality laws, and is a valuable backup storage method. It also allows for open comparison to other sites (e.g. Appalachia) in which VCOM students are logging their clinical experiences. Together, the two databases (the CREDO ICD Logger and the local spreadsheet) provide secure and reliable access to patients' information.

\section{RESUltS}

Data collection is completed every week; a designated doctor manually uploads the patients seen during the week from the

Published by Sciedu Press
HealthMaster database, the locally developed and established EMR system, to the CREDO ICD Logger.

The similarities and differences between two underserved sites are shown in Table 1. Tema is considered a suburban locality, with the most populous area being Community 1 . Appalachia was chosen because it is a socioeconomically disadvantaged population in the US; in some respects, it has similarities to a third-world country. Both sites have essential (primary) hypertension as their second most frequent diagnosis as well as disorders of lipoprotein metabolism and other lipidemias, which can be attributed to the increasing rate of obesity and lack of exercise. Similar drugs used to treat these conditions are frequently administered at both sites, such as Bendroflumethiazide, Hydrochlorothiazide, Atorvastin and Lisinopril. They both use various antibiotics, such as Amoxicillin, Ciprofloxacin and Azithromycin. However, the use of these drugs reflects the differences between diagnoses at the two sites. Although it is advanced, HMC has limited resources and cannot always obtain the best medications, limiting what prescriptions are available to the patients. Some of the diagnoses made in Ghana are respiratory in nature, possibly due to the working conditions, excessive dust in the environment and the plurality of viral infections in tropical environments. The prescriptions made to the patients in Appalachia are more orthopedic; for example, the diagnoses of dorsalgia and other joint disorders. Regular visitation of the doctor's office or "check-ups" (encounter for general examination without complaint) is not common in Ghana, due to the cultural standard of if one is not visibly ill, there is no need to go to the doctor.

\section{Discussion}

Most developing countries lack the technical expertise, funding and technological infrastructure needed for implementing EMR systems. ${ }^{[7]}$ However, some countries have proven that is possible to have a locally developed EMR, such as the one in Ghana. This EMR functions very well for the size of the clinic, although it does have technical issues with certain features. Using the CREDO ICD Logger supports this established system by providing a back-up on a server that is less likely to experience technical issues. It also provides a universal medical language (ICD codes) that enable worldwide comparisons. Due to its transparent and intuitive design and minimal cost, the implementation of the Logger at HMC would be beneficial for both parties (i.e., HMC and the research group at VCOM). We would have Sub-Saharan public health data at a per-patient resolution for future research and HMC would have a secure back-up of their patient information centrally located outside of the country. Transcribing data from one EMR system to another is an arduous and prolonged task. However, capturing universal information (ICD 
codes) for a patient and having a separate backup of patient information was considered valuable. After initial tests, we concluded that bi-weekly data conversion and collection was the best option.

Table 1. The Top 10 Diagnoses for the Ghana and Appalachia sites. Using the "Top 10" feature in the Logger page, these lists were composed to demonstrate the similarities and differences between the locations

\begin{tabular}{ll}
\hline Clinic Rotation at HMC & Family Medicine in Appalachia \\
\hline Top 10 Diagnoses & Top 10 Diagnoses \\
- Plasmodium falciparum malaria & - Encounter for general examination without complaint, suspected \\
- Essential (primary) hypertension & or reported diagnosis \\
- Other disorders of urinary system & - Essential (primary) hypertension \\
- Acute upper respiratory infections of multiple and unspecified sites & - Type 2 diabetes mellitus \\
- Other anemias & - Disorders of lipoprotein metabolism and other lipidemias \\
- Disorders of lipoprotein metabolism and other lipidemias & - Biomechanical lesions, not elsewhere classified \\
- Vasomotor and allergic rhinitis & - Dorsalgia \\
- Other and unspecified soft tissue disorders, not elsewhere classified & - Other joint disorder, not elsewhere classified \\
- Unspecified acute lower respiratory infection & - Persons encountering health services in other circumstances \\
- Open wound of head & - Encounter for screening of malignant neoplasms \\
& - Long term (current) drug therapy \\
Top 10 Prescriptions & Top 10 Prescriptions \\
- Ibuprofen & - Lisinopril \\
- Ciprofloxacin & - Levothryoxine sodium \\
- Cetirizine & - Amoxicillin \\
- Folic acid & - Gabapentin \\
- Metronidazole & - Dexamethasone \\
- Amoxicillin & - Atorvastatin \\
- Diazepam & - Hydrochlorothiazide \\
- Bendroflumethiazide & - Azithromycin \\
- Naproxen & - Prednisone \\
\hline
\end{tabular}

\section{Conclusions}

The CREDO ICD Logger system, as an ancillary EMR system was tested in a new hospital in Ghana, a developing nation, which enabled the hospital to have an off-site backup of critical medical records. Capturing the information in a universal way, using WHO's ICD-10 codes, enabled us to compare the public health picture in Ghana relative to a US population. This trial demonstrated the utility of the CREDO ICD Logger when used in conjunction with an established EMR system, but also showed it potential as a stand-alone EMR system, sufficient for developing nations, provided that it is accompanied with a database that relates the internal CREDO ICD Logger patient ID with their true ID. By keeping the conversion database local, confidential and encrypted it is possible to separate the bulk of the patient information via ICD-10 codes from the confidential information. This, in turn, demonstrates that a large portion of the information, information of particular value to continuously monitoring and surveilling public health down to individual anonymous patients is possible.

We have shown that the CREDO ICD Logger system can be used independently or to supplement existing EMR systems, enabling the merging and comparison via the universal ICD codes at different locations to evaluate the similarities and differences in general public health. We have also shown that by supplementing the CREDO ICD Logger with a local confidential encrypted patient name to CREDO ICD Logger internal patient ID that it has the potential to work as a hybrid stand-alone EMR system. Thus, the CREDO ICD Logger can be used in other developing countries with hospitals that lack an ample EMR system. By providing a centrally located system which can be used internationally, an EMR system such as the Logger can help shape and improve health care around the world.

\section{ACKNOWLEDGEMENTS}

We wish to thank the Bradley Foundation and the Edward Via College of Osteopathic Medicine internal fund for supporting this research. We also wish to thank all the medical students, staff and faculty of the Edward Via College of Osteopathic Medicine for feedback and contributions to this project.

\section{CONFlicts of InTERest Disclosure}

The authors declare they have no conflicts of interest. 


\section{REFERENCES}

[1] Garret P, Seidman J. EMR vs EHR - What is the Difference? Health IT Buzz. 2011.

[2] Meigs SL, Solomon M. Electronic Health Record Use a Bitter Pill for Many Physicians. Perspect Health Inf Manag. 2016; 13.

[3] HIT. Office-based Physician Electronic Health Record Adoption n.d. Accessed January 9, 2018. Available from: https://dashboard.healthit.gov/quickstats/pages/ph ysician-ehr-adoption-trends.php

[4] Boonstra A, Broekhuis M. Barriers to the acceptance of electronic medical records by physicians from systematic review to taxonomy and interventions. BMC Health Services Research. 2010; 10: 231. PMid: 20691097. https://doi.org/10.1186/1472-696 3-10-231

[5] Miller RH, Sim I. Physicians' use of electronic medical records: barriers and solutions. Health Affairs. 2004; 23: 116-126. PMid: 15046136. https://doi.org/10.1377/hlthaff .23.2.116

[6] Fraser H, Biondich P, Moodley D, et al. Implementing electronic medical record systems in developing countries. Journal of Innovation in Health Informatics. 2005; 13: 83-95. https ://doi .org/10 $.14236 / j h i . v 13 i 2.585$

[7] Williams F, Boren S. The role of the electronic medical record (EMR) in care delivery development in developing countries: a systematic re- view. Journal of Innovation in Health Informatics. 2008; 16: 139-45. https://doi.org/10.14236/jhi.v16i2.685

[8] Sood SP, Nwabueze SN, Mbarika VW, et al. Electronic medical records: A review comparing the challenges in developed and developing countries. Hawaii International Conference on System Sciences. 2008: 248. https://doi.org/10.1109/HICSS. 2008.1 41

[9] Luk R, Ho M, Aoki PM. Asynchronous Remote Medical Consultation for Ghana. 2008; 743.

[10] World Health Organization. International statistical classification of diseases and related health problems: Tenth revision. 2016; 2(2): 1-169. Available from: http://apps.who.int/iris/bitstrea $\mathrm{m} / 10665 / 42980 / 1 / 9241546530 \_$eng.pdf

[11] Rawlins F, Sumpter C, Sutphin D, et al. Quantifying medical student clinical experiences via an ICD Code Logging App. International Journal of Medical Informatics. 2018; 111: 51-57. https : //doi.org/10.1016/j.ijmedinf.2017.12.017

[12] Doan P, Oduro CY. Patterns of Population Growth in Peri-Urban Accra, Ghana. International Journal of Urban and Regional Research. 2012; 36: 1306-1325. https://doi.org/10.1111/j.1468-242 7.2011.01075.x

[13] Ghana Wellness Foundation. 2016. Available from: https ://www . gwfgh.org 